\title{
Study on driving the development of large data innovation and Zhuhai Jin Wan
}

\author{
Ying $\mathrm{LI}^{1}$, \\ ${ }^{1}$ Guangdong Polytechnic of Science and Technology, Zhuhai,Guangdong.519090
}

Keywords: Big data; Innovation; Drive

\begin{abstract}
New period, Big data is the national basic strategic resources, Has become a new element of social production, is the new oil, new assets, new space, is a profound change in our science and technology, industry and management. The big data technology is bringing a revolution, big data not only means that the mass, diversity, fast data processing, it is a new factor of production. An innovative resource and a new way of thinking. By discussing how to use data resources and change the mode of economic growth in Zhuhai jinwan, Zhuhai jin wan drive to boost the development of innovation, so as to serve the enterprise decision-making and governance, so that we understand, is an important strategic issue worthy of our new thinking to use big data.
\end{abstract}

\section{Introduction}

\section{The current situation of the development of big data driven and Zhuhai jinwan innovation}

Big data development status. Big data, or a huge amount of data, massive data; is a huge number, complex structure, many types of data consists of data collection ${ }^{[1]}$, data processing and application model based on cloud computing, through data integration and sharing, intellectual resources and knowledge service capability of cross formation. There are research institutions so definition of big data: is the need for new data processing mode to have a stronger decision-making ability, insight discovery and process optimization capabilities of massive, high rates of growth and diversification of information assets. To some extent, big data is a cutting-edge technology of data analysis. In short, from various types of data, ability to quickly obtain valuable information, is the big data technology.

Zhuhai Jinwan innovation driven development. Jinwan District was founded 16 years ago, ${ }^{[3]}$ the district government has been committed to creating an important gathering area of high-end industrial development in Zhuhai, an important window to show the internationalization of the image of the city, the core area of Western Ecological City, ${ }^{[2]}$ the "three position", "ten industrial projects", "ten people's livelihood project", "five the development of platform construction as the starting point, to speed up the development of the three strategic emerging industries, accelerate industrial restructuring and upgrading, and launched the" two business "activities. The Bay Area pioneered the development in the reform, innovation in development, and always adhere to the development and innovation as the lifeline of the soul.

At present, the jinwan formed with enterprises as the driving system of independent innovation, the federal pharmaceutical, Livzon pharmaceutical, PHILPS, TDK, caiga, cirrus and a number of innovative enterprises in the rapid rise of LED, Guangdong Institute of Science and Technology, Zunyi Medical College and other five universities in Zhuhai, Institute of biomedicine, Aviation Technology Research Institute and other research institutions in the the bay is established. The bay area to encourage innovation in the environment of innovation and financial innovation, tolerance of failure, a strong cultural atmosphere, the power of innovation, innovation, innovation and stimulate potential mining. The market plays a fundamental role in the allocation of innovation resources and give full play to the level of industrial innovation in the leading position, the effective implementation of intellectual property rights the protection and standardization strategy, and constantly improve the innovation elements, innovation service system gradually perfect. 


\section{Zhuhai Jinwan analysis of innovation driven development advantages}

\section{Zhuhai Jinwan advantage and innovation driven development.}

Important strategic position.As the reform and opening up the window and Zhuhai jinwan comprehensive reform pilot area, and always adhere to the development and innovation as the lifeline of the soul, has become the Zhuhai high tech industrial base and property demonstration area of knowledge, is to promote Zhuhai to become an important force in the construction of innovative city.2015 years, Jinwan District conscientiously implement the innovation driven development strategy to major projects as the starting point, to promote the rapid development of advanced equipment manufacturing industry, to accelerate the pace of industrial transformation and upgrading. 3 the first half of 2015, the bay area is expected to achieve GDP 8 billion 636 million yuan, an increase of 7.5\%; investment in fixed assets 4 billion 498 million yuan, an increase of $10.7 \%$.

Strong economic strength. Since its establishment, the jinwan sustained and rapid economic development, GDP are also on the rise, the growth rate of GDP except in 2013 was higher than that of Zhuhai GDP growth.2014 region GDP exceeded 20 billion yuan, an increase of 11.7\%; per capita GDP exceeded 130 thousand yuan; above scale industrial output value reached 52 billion 101 million yuan, an increase of 9.1\%; completed investment in fixed assets 10 billion 865 million yuan, an increase of 21.8\%; the actual use of foreign direct investment with an increase of $11.8 \%$; the public budget revenue 1 billion 866 million yuan.

Superior innovation environment. The jinwan formed an independent innovation system with enterprises as the main body. Firstly, strengthen the base of innovation in Jinwan enterprises as the core, set up the annual investment of not less than 100 million yuan of industrial innovation driven development funds to support the new R \& D institutions, construction of public service platform and incubator innovation platform. At the same time, industrial support above scale enterprises to establish enterprise technology center and other research institutions, especially focusing on the franc, the federal, provincial and municipal engineering center, China Resources, the construction of enterprise technology center. Secondly, another magic Jinwan District to enhance innovation drive level. Since this year, Jinwan District innovative investment The way, aiming at the industrial base, domestic and foreign well-known enterprises, scientific research institutions, service platform, to create a professional investment team, the recruitment of overseas investment agency in professional organizations at home and abroad. At the same time, the main leadership often led to foreign investment in Jinwan District, has been to the United States, Canada, Beijing, Chongqing and other places, to promote aviation industrial park, Lianzhou large general aviation, medical equipment and other key projects to accelerate the construction of settled. Therefore, innovation policy environment improved significantly.

\section{Zhuhai Jinwan disadvantages in innovation driven development}

Innovation of high and new technology enterprises needs to be improved. After years of development, the initial formation of the jinwan with enterprises as the main body of independent innovation system in.2014 years, the Bay Area in the above scale industrial enterprises reached 216, 14 billion 600 million yuan of industrial added value, above scale industrial added value accounted for $73.94 \%$ of GDP, the above scale industrial enterprises realized a total profit of 3 billion 630 million yuan, an increase of 35.09\%., Jinwan District has 55 high-tech enterprises, the output value of about 25000000000 yuan, accounting for above scale industrial output value of about 50\% local; with aviation, biomedicine two national new industrialization demonstration base, [3] provincial base 22, level 30. the city's Administrative Region ranked first. But with the rapid development of enterprise innovation ability under the background, there are still the most high-tech enterprise innovation ability is not strong, especially in biological engineering and The new energy industry is the most obvious. There are three reasons: one. As enterprise technical support and technology incubator and has strong research and development strength of universities and research institutions are too few; two. The majority of Jinwan high-tech enterprises are small, scattered strength in Scientific Research Investment Co.; three. A considerable part of the bay the technology is imported from overseas after the "two development" or directly used for the production of new products, new 
technology developed less.

Lack of core technologies and cutting-edge technologies.High tech enterprises in Jinwan foreign investment, but foreign investment in production areas, and to invest in technology research and product development is relatively small. Most foreign to Jinwan as its production base and assembly base, such as intelligent robot of new energy industry output value of $2 / 3$, the advanced technology of great value basically in the hands. A few large multinational companies, but the meager profits. Despite the high-tech industry also put money and effort in the development of enterprises, but the core technology is still dependent on technology introduction, technology purchase, "market for technology" strategy is not for Jinwan high-tech industry the core competitiveness, the independent innovation is the fundamental way to the development of the industry.

There are drawbacks in the pattern of innovation investment. R\&D $90 \%$ jinwan located at the enterprise, $85 \%$ technology developers focus on the enterprises, $80 \%$ of the R\&D funds from enterprises ${ }^{[3]}$, Enterprises and enterprise groups become the subject of Jinwan high-tech industry investment, this pattern has many advantages, but also have significant drawbacks. Because the enterprises as the main business of the Bay will put a lot of resources development hides a high risk of failure, once the development will bring huge losses. Innovation is a complex process of learning.

\section{Big data driven architecture development assistance Jinwan innovation}

Jinwan skillfully make use of large data help drive innovation development. At present, the rapid development of information technology, information technology innovation emerge in an endless stream, [2] "big data" is the leading information wave is sweeping across the Bay, the Bay become the enterprise to accelerate technological innovation, promote the transformation of government functions, a leading tool social change management. The Bay imformational sustained and rapid development and wide application of information technology in the field of the economy and society, information resources collection, analysis and exchange system is becoming more and more deeply. The value of mining scale Jinwan e-government information resources sharing and exchange platform, covering a number of government departments, sharing data over the Internet, almost Dayi. But in the face of large numbers According to the development of new challenges, Jinwan still exists many problems, mainly in the "information island" phenomenon in the sharing of data resources is not high, the small data size leads to the value of the data mining application, data service is still at the initial stage led to the emergence of no key breakthrough related core technologies and business models.

\section{The framework of big data innovation power drive Jinwan.}

The era of big data, how to the use of data resources to change the mode of economic growth, drive to boost the development of the service in Jinwan innovation, enterprise decision-making and governance, is an important strategic issue worth pondering. In Jinwan embrace big data at the same time, ${ }^{[2]}$ can "frame the development and utilization of cloud - chain - end" good (chart 1 for "cloud chain the relationship between the three - end"). "Cloud computing," chain "construction and" end of innovation ", make big data truly to bring Jinwan big industry, big opportunities and big bonuses.

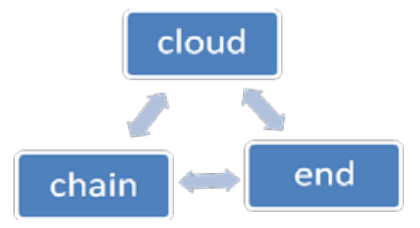

Fig. 1 Big data development framework

The "Cloud" computing. Cloud is the processing center of big data, cloud computing can effective integration of information technology and industrialization of Golden Bay, make production efficiency has been greatly improved. The bay is a cloud, Jinwan industrial era big data into the Internet era of big data. Cloud computing and big data is like the two wheels of the car and 
the bird the two wings: cloud computing is the driving force of the bay big data development, big data need to implement cloud computing solutions. All kinds of data not only reflects the objective state of Jinwan things, also contain the law of development of things. This rule the Bay Development Bay of the whole society, we once mastered, can grasp the pulse of the development of the jinwan even predict Jinwan future. From a global perspective, "quantitative decision" and "data governance" has become represent the general trend. At present, we should learn from the "qualitative in Jinwan dream journey "To the" combination of qualitative and quantitative, set up based on the data, facts and rational analysis of the management concept.

The "chain" construction. The chain is a big data infrastructure, should construct a link to create a cloud to cloud, cloud to end, end to end interoperability, to achieve different levels of Jinwan different application fields of data sharing and efficient use of big data link. As with interaction, intelligent network relay transmission, can be integrated in the bay "information island" and "application island", so that each network data terminal Jinwan incarnation battlefield commander. Today, the data has become like energy, mineral resources as a strategic, followed by data security and privacy issues, especially the need to pay attention to the "Golden Bay joint defense cloud chain - end" to avoid. The risk of data leakage, the Bay should be based on big data technology and use of domestic platform, actively support and guide the local enterprises to increase research and development efforts, and strive to break through the core technology, and gradually improve the key facilities of self-control Level. At the same time, but also the data ownership bay "and" data privacy regulations or standards to protect local citizens and government units of data security by law.

The "end" is the end of innovation. The innovation direction in Jinwan big data, to the data collection methods, innovation Bay terminal Quweicunzhen, multi angle credibility validation data; and as far as possible will Jinwan data open to the terminal, promote the innovative application of Jinwan terminal. From the market point of view, Jinwan should develop the intelligent terminal, to explore new business model; as for the government, should Jinwan smart city construction, promote the modernization of national governance. At present, the main reason is that the enterprise cannot reach the Jinwan big data applications, no interaction between excitation data and business scene. Enterprises should make Jinwan demand and technology of real-time, dynamic, economic docking, users become big data providers and beneficiaries the realization of iterative closed-loop operation operation and use. Compared with enterprise, Jinwan government has a natural advantage in the data, not only acts as the data "accountant". Jinwan government should wake up sleeping in the portfolio, the valid data in memory, and provide a reliable basis for their scientific policy and rational allocation of resources.

\section{Concluding remarks}

This article from the current situation of the development of big data, through the analysis of Zhuhai Bay innovation drive development advantages and disadvantages, and the era of big data, how to use the jinwan, the use of big data boost innovation and development framework of Jinwan, big data boost Jinwan innovative "cloud chain end". Summed up in the era of big data, the data is wealth is a resource, is the competitiveness. The state of the data is not only a massive big data and the corresponding data processing technology, it is a new way of thinking, is a series of new ideas, new methods, and focuses on the new elements.

\section{References}

[1]Ding Shengyong, Min Shiwu, pan Yong Bing, doubts. Big data M. The posts and Telecommunications Press, 2013:1

[2]Zhao Guodong, Yi Huan Huan, MI Wan Jun, Hubei vilane. The era of big data opportunities M. Tsinghua University press, 2013:9

[3]Tao Renji. Cryptography and Mathematics (J). Journal of nature, 1984,7 (7):527 